\title{
Puerarin protects against damage to spatial learning and memory ability in mice with chronic alcohol poisoning
}

\author{
S.Q. Cui ${ }^{1}$, Q. Wang ${ }^{2}$, Y. Zheng ${ }^{2}$, B. Xiao ${ }^{2}$, H.W. Sun ${ }^{1}$, X.L. Gu ${ }^{1}$, Y.C. Zhang ${ }^{1}$, C.H. Fu ${ }^{1}$ \\ P.X. Dong ${ }^{1}$ and X.M. Wang ${ }^{2}$ \\ ${ }^{1}$ China Shandong Provincial Engineering Laboratory of New Pharmaceutical Excipients, \\ Sustained and Controlled Release Technology, College of Medicine and Nursing, Dezhou University, Dezhou, China \\ ${ }^{2}$ Department of Physiology, Capital Medical University, Beijing, China
}

\begin{abstract}
We evaluated the effect of puerarin on spatial learning and memory ability of mice with chronic alcohol poisoning. A total of 30 male C57BL/6 mice were randomly divided into model, puerarin, and control groups ( $\mathrm{n}=10$ each). The model group received $60 \%(\mathrm{v} / \mathrm{v})$ ethanol by intragastric administration followed by intraperitoneal injection of normal saline 30 min later. The puerarin group received intragastric $60 \%$ ethanol followed by intraperitoneal puerarin 30 min later, and the control group received intragastric saline followed by intraperitoneal saline. Six weeks after treatment, the Morris water maze and Tru Scan behavioral tests and immunofluorescence staining of cerebral cortex and hippocampal neurons (by Neu-N) and microglia (by lb1) were conducted. Glutamic acid (Glu) and gamma amino butyric acid (GABA) in the cortex and hippocampus were assayed by highperformance liquid chromatography (HPLC), and tumor necrosis factor (TNF)- $\alpha$ and interleukin (IL)-1 $\beta$ were determined by ELISA. Compared with mice in the control group, escape latency and distance were prolonged, and spontaneous movement distance was shortened $(\mathrm{P}<0.05)$ by puerarin. The number of microglia was increased in both the cortex and hippocampal dentate gyrus $(P<0.01)$, and neurons were reduced only in the hippocampal dentate gyrus $(P<0.01)$ in puerarin-treated mice. In the model group, Glu and GABA levels decreased $(P<0.05)$, and Glu/GABA, TNF- $\alpha$, and IL-1 $\beta$ increased $(P<0.01)$ with puerarin treatment, returning to near normal levels. In conclusion, puerarin protected against the effects of chronic alcohol poisoning on spatial learning and memory ability primarily because of anti-inflammatory activity and regulation of the balance of Glu and GABA.
\end{abstract}

Key words: Puerarin; Chronic alcohol poisoning; Neuroprotection; Microglia; Inflammatory factors

\section{Introduction}

Chronic alcoholism is a serious social problem that can cause pathological changes in the structure and function of the central nervous system (CNS), resulting in inattention, cognitive dysfunction and poor judgment, unstable walking, and even dementia (1-6). The heavy burden of alcohol has made it a global medical and social problem (7). Reactive oxygen species are thought to contribute to many neurodegenerative diseases, such as Alzheimer's or Parkinson's disease, as well as Down syndrome (8-11). However, a recent study did not find a relationship between acute ethanol-induced memory deficit and oxidative stress (12). Moreover, it has been reported that treadmill running can attenuate the adverse effects of chronic ethanol exposure on spatial memory (13). However, compliance with running training is poor. Therefore, there is still no satisfactory treatment of the nervous system impairment caused by long-term intake of alcohol.
Puerarin is one of the main active ingredients of the traditional Chinese herb, Radix Puerariae lobata, and is known chemically as 8-beta-D-grapes pyranose-4',7 dihydroxy isoflavones $\left(4^{\prime}, 7\right.$ dihydroxy-8-beta-D-glucosylisoflavone). The powder has long been used as an anti-alcoholic drug. Puerarin can easily go through the blood brain barrier into the CNS. It is reported that puerarin flavonoids improve the blood circulation in the brain and heart, counter memory disorders (14), protect nerve cells against glutamate injury, and alleviate damage of astrocytes $(15,16)$. Puerarin has many pharmacokinetic advantages, such as a short halflife, rapid elimination, and not easily being accumulated in the body (17), all of which make it a suitable candidate for clinical use as a medication. It has been widely used to treat cardiovascular diseases of humans and animals (18-20). However, the protective role of puerarin on ethanol-induced impairment of the CNS has not been reported.

Correspondence: Xiaomin Wang: <xiaominwangxw@163.com> and/or Hanwen Sun: <hanwen916@163.com>. 
In this study, we induced chronic alcohol poisoning in a mouse model and investigated the ability of puerarin to protect against alcohol-related damage of spatial learning ability and memory. Effects of alcohol and puerarin on cerebral neurons, glial cells, and related neurotransmitters are described and a mechanism of protection proposed.

\section{Material and Methods}

\section{Animals and treatment}

All procedures were approved by the Ethics Committee of Dezhou University, China. A total of 30 healthy male C57BL/6 mice of 8-10 weeks of age and weighing 18-22 g were supplied by the Vital River Laboratory Animal Technology Co., Ltd. (China). Mice were housed in an airconditioned facility at an ambient temperature of $22 \pm 2{ }^{\circ} \mathrm{C}$, a relative humidity of $40 \%-60 \%$, and a 12-h (7:00 am to 7:00 pm) light/dark cycle. They were fed a standard laboratory diet and distilled water ad libitum. After 1 week of acclimation, the animals were randomly divided into three groups of 10 animals each. The model group was given $60 \%(\mathrm{v} / \mathrm{v})$ ethanol by intragastric administration $(6 \mathrm{~mL} / \mathrm{g}$ daily in the first week, $12 \mathrm{~mL} / \mathrm{g}$ daily in the second and third weeks, and $20 \mathrm{~mL} / \mathrm{g}$ daily in the following 3 weeks) followed by intraperitoneal injection of normal saline. In the first 3 weeks, ethanol was administered once daily at 10:00 am; in the following 3 weeks, it was administered twice daily at 10:00 am and 4:00 p.m. The puerarin group received the same treatment as the model group, except that normal saline was replaced by intraperitoneal administration of puerarin solution. Mice received $100 \mathrm{mg} / \mathrm{kg}$ puerarin daily in the first week, $150 \mathrm{mg} / \mathrm{kg}$ daily in the second and third weeks, and $20 \mathrm{mg} / \mathrm{kg}$ daily in the following 3 weeks. The control group was treated with normal saline by intragastric administration and intraperitoneal injection. In all three groups, the intraperitoneal injection was administered once daily at about 10:30 am, 30 min after the intragastric treatment. Puerarin for injection was obtained from Zhejiang CONBA Pharmaceutical Co., Ltd. (approval no. H33020186, China) and anhydrous ethanol was provided by Shanghai Chemical Reagents Co., Ltd., reagent no. 2 factory (China). During the experimental procedures, all animals had free access to food and distilled water ad libitum.

\section{Behavioral tests}

The Morris water maze (MWM) and Tru Scan field activity tests were carried out on the day following the 6 weeks of treatment.

Tru Scan field activity testing. This test was performed from approximately 9:00 to 11:00 am for 3 days after completion of the experimental intervention. To adapt to the environment, mice were moved to the behavioral science laboratory $30 \mathrm{~min}$ before testing. Individual mice were then transferred to the middle of the Tru Scan test box and the door was closed. Under quiet experimental conditions, the distance and trajectory of spontaneous motion of the mice were recorded using the infrared detector of the Tru Scan Photobeam Activity System (Coulbourn Instruments, USA). The box was cleaned with $10 \%$ ethanol to avoid any influence of smell of the previously tested mouse.

Morris water maze. The MWM behavior test was conducted from about 14:00-16:30 p.m. as previously described (21). This test was used to assess the spatial learning and memory ability of the experimental animals. The maze consisted of a circular tank $(1.50 \mathrm{~m}$ in diameter and $0.6 \mathrm{~m}$ deep) with black walls. The tank was filled with water maintained at a temperature of $25 \pm 1^{\circ} \mathrm{C}$. The pathtracking and recording system was provided by Actimetrics (Actimetrics, Inc., version 3.41, USA). Testing was carried out over 6 days, with the first day regarded as screening for basic athletic ability. A small, black platform about $20 \mathrm{~cm}$ in diameter was placed in a quadrant of the tank so that it extended $1 \mathrm{~cm}$ above the water. Mice from each group were placed individually in the water maze in the same quadrant, not the one with the platform, and the times it took to find the platform were recorded and compared to evaluate vision and swimming ability. For spatial navigation training and testing, the platform was placed $1 \mathrm{~cm}$ underwater in a different quadrant; mice underwent the same treatment as on the first day and were required to find the hidden platform in $2 \mathrm{~min}$. This test evaluated spatial learning ability by recording and analyzing the escape latency (i.e., time to reach the platform) and escape distance (i.e., length of the swimming path taken to find the platform). In the spatial probe trial that was conducted on the last day, mice underwent the same treatment as in previous days, but the platform was removed and time was controlled at $1 \mathrm{~min}$. The crossing times (i.e., the number of times the mice passed through the platform position) and total swimming distance in the controlled time were recorded and compared; the escape route (i.e., the swimming path) was recorded to evaluate the escape strategy.

\section{Immunohistochemical detection of Neu-N and Iba-1}

After behavioral testing, three mice from each group were anesthetized with pentobarbital sodium $(80 \mathrm{mg} / \mathrm{kg}$ ) and underwent cardiac perfusion with physiological saline to obtain clean brain tissue without blood. The dissected tissue was fixed in $4 \%$ paraformaldehyde for more than $24 \mathrm{~h}$, frozen, sectioned at $10 \mu \mathrm{m}$, and mounted on slides. After antigen retrieval and blocking of endogenous peroxidase activity, sections were incubated with monoclonal mouse anti-neuronal nuclei antibody (Neu-N, 1:500, Chemicon, USA) or rabbit-anti-mouse lba-1 antibody (1:500, Wako Chemicals, Japan) at $4{ }^{\circ} \mathrm{C}$ overnight to detect brain neurons and microglia. After that, the sections were incubated with goat anti-mouse IgG labeled with red-fluorescent Alexa Fluor 594 (Molecular Probes, USA) or goat-anti-rabbit IgG labeled with green-fluorescent Alexa Fluor 488 (Molecular Probes) secondary antibodies. At least 10 serial sections from the hippocampus and cortex of three mice were 
selected, viewed, and photographed using a laser confocal scanning microscope (Leica, Germany). The numbers of microglial cells and neurons in the cortex were counted during microscopic observation, and those in the hippocampal dentate gyrus were calculated from the fluorescence intensity using Image-Pro Plus 6.0 (Media Cybernetics, USA).

\section{Assay of Glu and GABA by high-performance liquid} chromatography (HPLC)

The remaining mice in each group were sacrificed, the brain cortex and hippocampus were quickly isolated, frozen separately in liquid nitrogen, and stored at $-80^{\circ} \mathrm{C}$ until use. For the assays, the stored cortex and hippocampus were thawed and then homogenized with $4 \%$ sulfosalicylic acid. Then, the content of Glu and GABA in the homogenate was assayed by HPLC (ESA, USA) and the Glu/GABA ratio was calculated. The Glu and GABA standards were purchased from Sigma (USA).

\section{Assay of TNF- $\alpha$ and IL-1 $\beta$ by ELISA}

Stored cortex and hippocampus were thawed and homogenized. TNF- $\alpha$ and IL-1 $\beta$ in the homogenates were measured using commercial ELISA kits (Excell Company, USA).

\section{Statistical analysis}

Data were analyzed using the Prism 5.0 software (GraphPad Software, USA). Data are reported as means \pm SE. One-way analysis of variance (ANOVA) was used to compare means within groups. A $P$ value $<0.05$ was considered to be statistically significant.

\section{Results}

Puerarin improved movement disorders in mice with chronic alcohol poisoning

The Tru Scan results revealed that the distance covered during spontaneous movement for 30 min was shorter in the model group than in the control group. The distance covered was significantly longer in the puerarin group than in the model group, as shown in Figure $1(\mathrm{P}<0.05)$.

Puerarin improved the spatial learning memory disorder of mice with chronic alcohol poisoning. In the spatial navigation test, the escape latency and escape distance of the model group were significantly longer than in the control group $(\mathrm{P}<0.05$ and $\mathrm{P}<0.01$, respectively; Figure $2 \mathrm{~A}$ and $\mathrm{B})$. When treated with puerarin, the escape latency on days 3,4 , and 5 and the escape distance on days 3 and 5 days in the puerarin group were significantly reduced compared with the control group $(\mathrm{P}<0.05$ and $\mathrm{P}<0.01$, respectively). In the spatial probe trial, the cross times and total swimming distance of the model group were significantly shorter than those in the control group, but in the puerarin group, the cross times and total swimming distance were similar to those in the control group and significantly longer than those in the model group (Figure $2 \mathrm{C}$ and $\mathrm{D}, \mathrm{P}<0.05$ ). The escape routes might reflect the escape strategy of mice. As shown in Figure 2E, the escape route of the mice in the control group was short and direct, while in the model group, the route was complex and wandering. In the puerarin group, the route was better than that in the model group, but still worse than in the control group.

\section{Influence of puerarin on the number of neurons and microglial cells}

As shown in Figures 3 and 4, there were significantly more microglial cells in the hippocampal dentate gyrus of the model and puerarin groups $(P<0.01)$, as well as in the cortex $(\mathrm{P}<0.05$ and $\mathrm{P}<0.01$, respectively) compared with the control group. Moreover, puerarin treatment inhibited the reduction of microglial cells in both the cortex $(P<0.05)$ and hippocampal dentate gyrus $(P<0.01)$ that occurred in the model group. The number of neurons was reduced only in the hippocampal dentate gyrus $(\mathrm{P}<0.05, \mathrm{P}<0.01)$.

\section{Influence of puerarin on Glu and GABA}

Compared with the control group, the Glu and GABA levels of the cortex and hippocampus were significantly

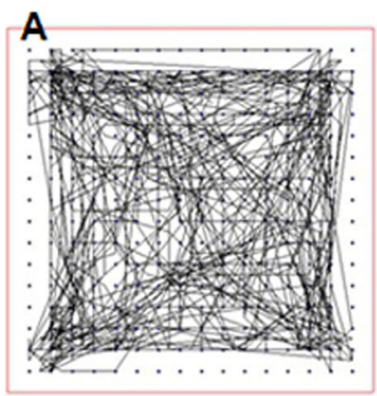

Control

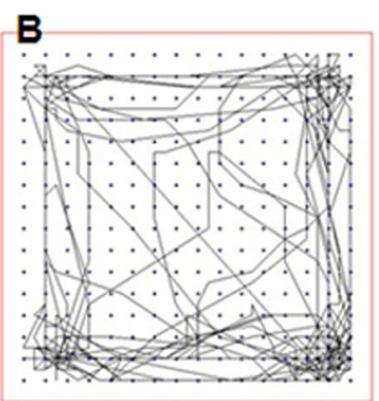

Model

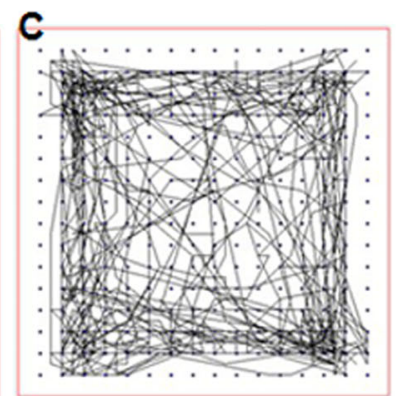

Puerarin

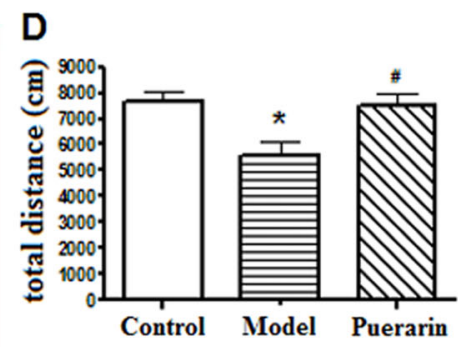

Figure 1. Results of the $30 \mathrm{~min}$ Tru Scan test. $A-C$, Spontaneous movement routes of mice in the control, model, and puerarin groups. $D$, Comparison of movement distance in 30 min among the 3 groups. ${ }^{*} \mathrm{P}<0.05$ vs control group; ${ }^{\#} \mathrm{P}<0.05$ vs model group (one-way ANOVA). 

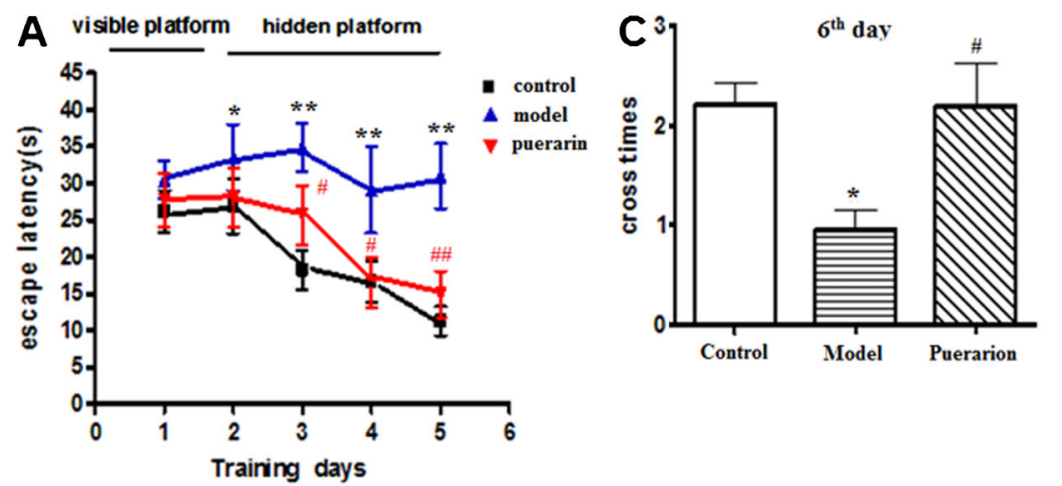

E

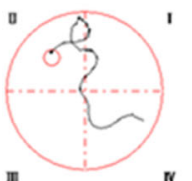

Control
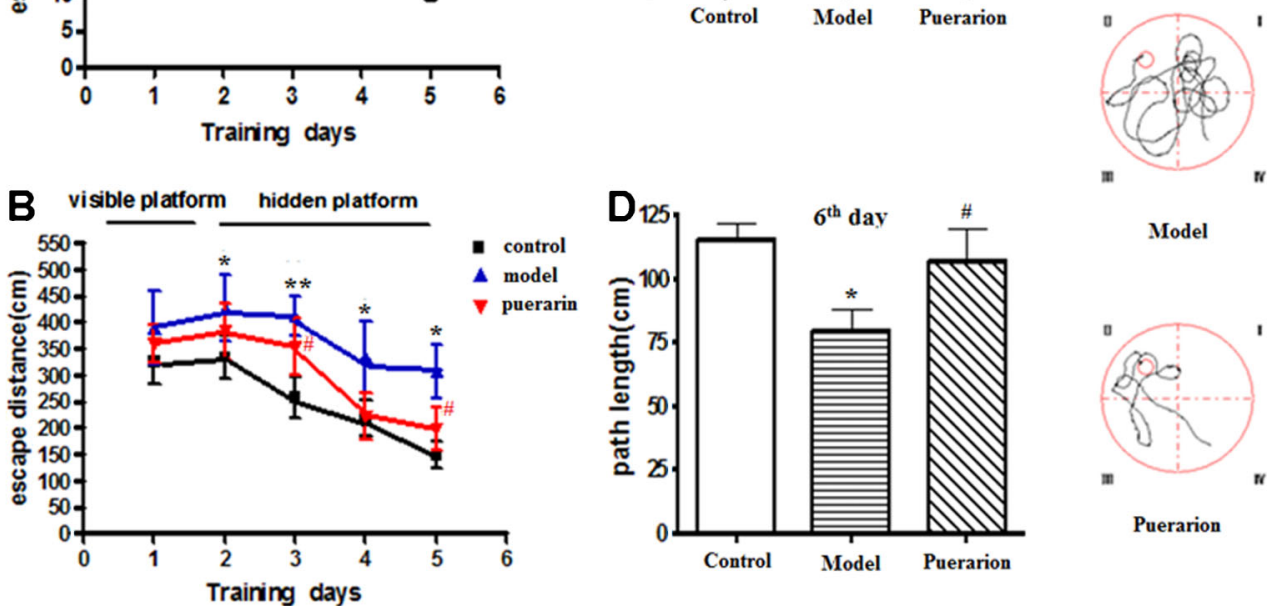

Model

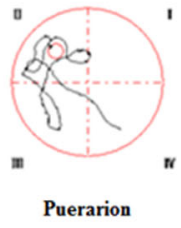

Figure 2. Results of the Morris water maze test. $A$, Escape latency: time to reach the hidden platform. $B$, Escape distance: length of the swimming path taken to find the platform. $C$, Cross times: times the mice passed through the platform. $D$, Total swimming distance in controlled time $(1 \mathrm{~min})$. E, Typical escape route (the swimming path of mice) in the 3 groups. ${ }^{*} \mathrm{P}<0.05$, ${ }^{* *} \mathrm{P}<0.01$ vs control group; ${ }^{\#} \mathrm{P}<0.05,{ }^{\# \#} \mathrm{P}<0.01$ vs model group (one-way ANOVA).
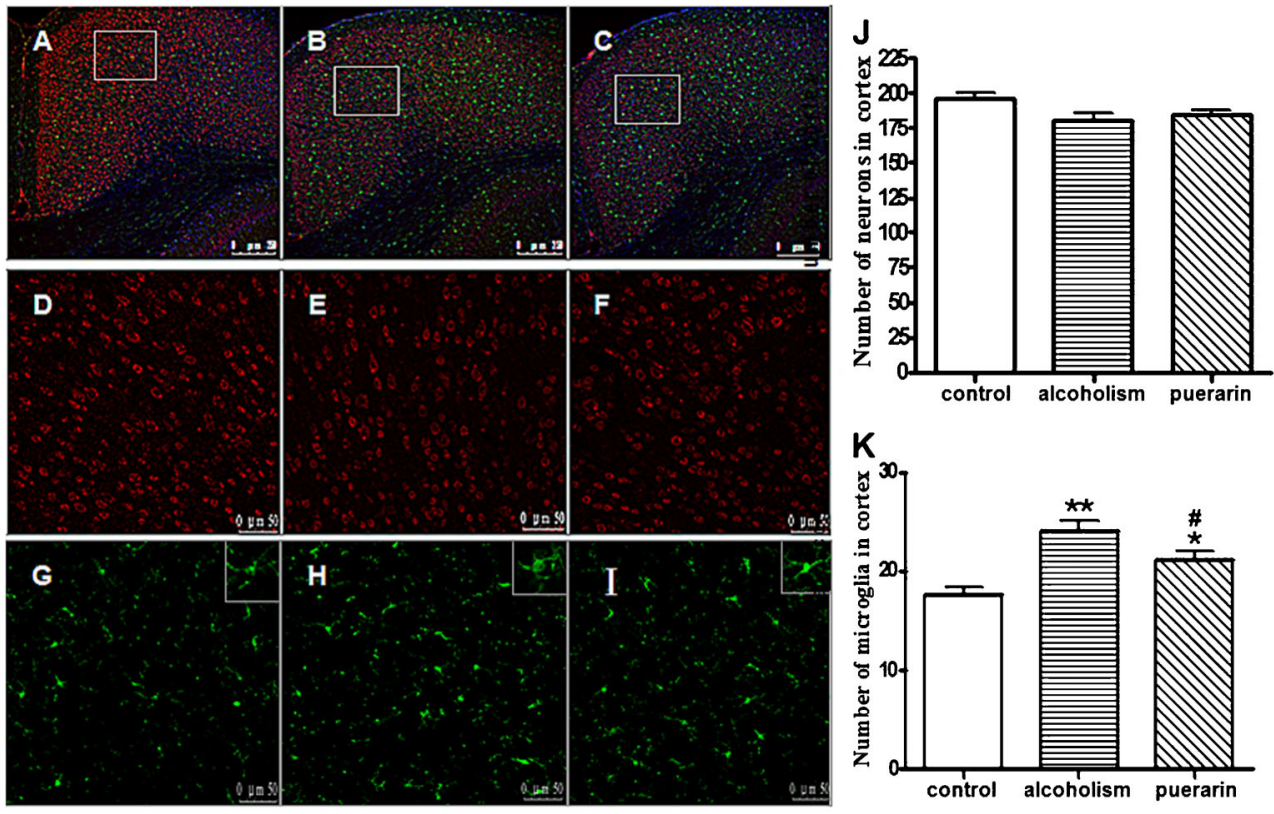

Figure 3. Detection of neuron and microglial cells in cortex of mice by immunohistochemical staining. A-C, Iba-1 immunofluorescence staining in the cortex of the control, model and puerarin groups $(10 \times)$. D-F, Cortical neurons of each group. G-I, Cortical microglia of each group. $J$, Number of neurons in the cortex of each group. $K$, Number of microglia in the cortex of each group. ${ }^{*} P<0.05$, ** $P<0.01$ vs control group; ${ }^{\#} \mathrm{P}<0.05$ vs model group (one-way ANOVA). 

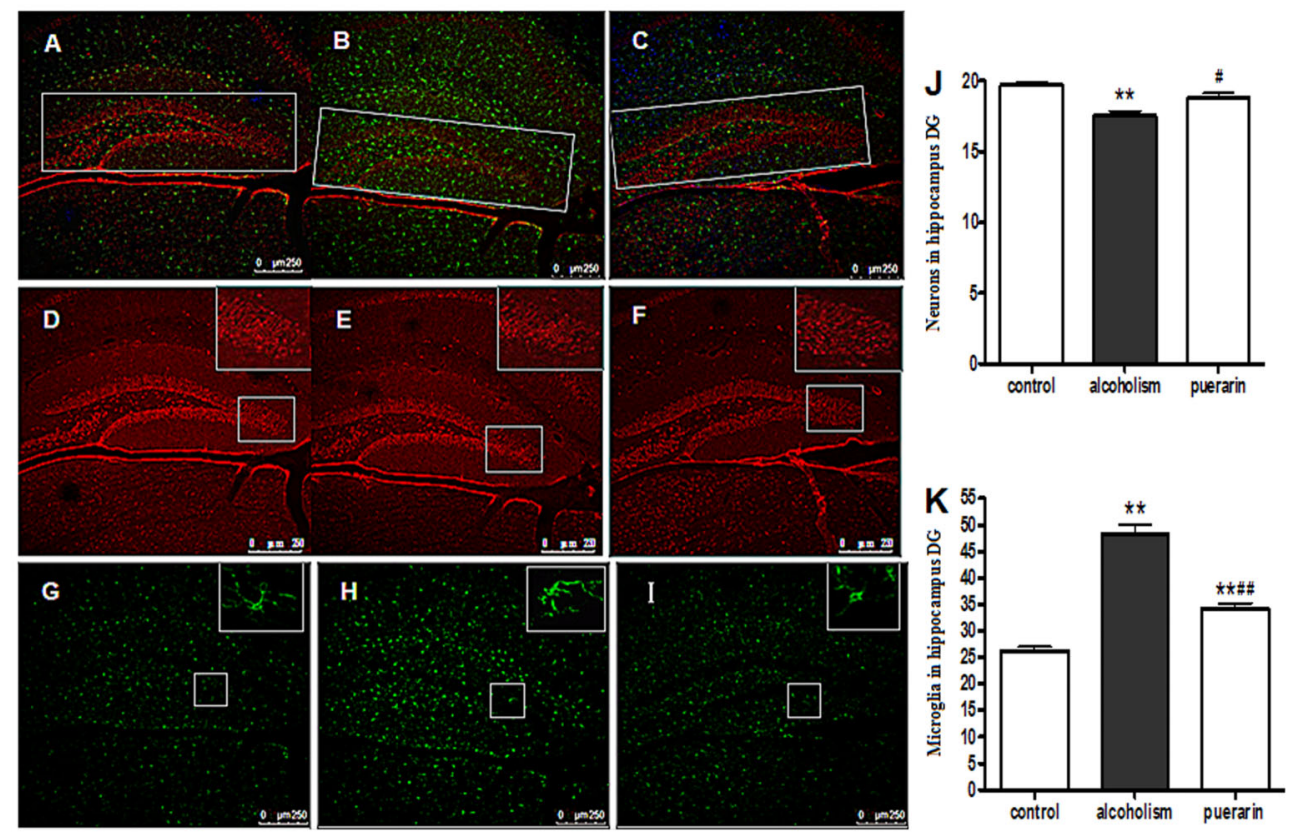

Figure 4. Detection of neuron and microglia cells in the hippocampus of mice by immunohistochemical staining. $A-C, \mathrm{Neu}-\mathrm{N}$ and Iba-1 immunofluorescence staining in the hippocampus of the control, model, and puerarin groups $(10 \times)$. $D-F$, Hippocampal neurons of each group. G-I, Hippocampal microglia of each group. J, Number of neurons in the hippocampus of each group. K, Number of microglia cells in the hippocampus of each group. DG: dentate gyrus. ${ }^{* *} \mathrm{P}<0.01$ vs control group; ${ }^{\#} \mathrm{P}<0.05$, ${ }^{\# \#} \mathrm{P}<0.01$ vs model group (one-way ANOVA).

reduced in the model group (Figure $5, \mathrm{P}<0.05$ and $\mathrm{P}<0.01$, respectively). Puerarin treatment significantly reversed the reduction of GABA in both the cortex $(P<0.05)$ and hippocampus $(P<0.01)$, but reversed only the reduction of Glu in the hippocampus $(P<0.05)$. The Glu/GABA ratio in the cortex was significantly higher in the model group than in both the control group $(P<0.05)$ and the puerarin group $(P<0.01)$. Puerarin treatment significantly inhibited the increase of the Glu/GABA ratio in the hippocampus compared with the model group $(P<0.01)$.

\section{Influence of puerarin on TNF- $\alpha$ and IL-1 $\beta$ in the cortex and hippocampus}

As shown in Figure 6, TNF- $\alpha$ and IL-1 $\beta$ in the cortex and hippocampus was significantly higher in the model group than in the control group, $(P<0.05$ and $P<0.01$, respectively). However in the puerarin group, TNF- $\alpha$ and IL-1 $\beta$ were significantly increased only in the hippocampus $(\mathrm{P}<0.05$ and $\mathrm{P}<0.01$, respectively). Puerarin treatment reversed the increase of TNF- $\alpha$ and IL-1 $\beta$ in both the cortex and the hippocampus compared with the model group $(\mathrm{P}<0.05)$.

\section{Discussion}

Alcoholism is usually caused by alcohol dependence and is reported to involve about 140 million people worldwide $(22,23)$. Animal models of alcohol poisoning are commonly established by feeding, gavage, or injection of alcoholic materials. In this study, we chose the gavage model (24), which is close to the situation in human beings. Moreover, the gavage model avoids differences caused by individual factors, such as appetite and food intake and thus more accurately reflects the impact of alcohol on the human body and the inhibitory effect of puerarin. In this study, blood alcohol concentration (BAC) reached $79.6 \mathrm{mg} / 100 \mathrm{~mL} 1 \mathrm{~h}$ after administration of $60 \%$ alcohol $(\mathrm{v} / \mathrm{v}, 6 \mathrm{~mL} / \mathrm{kg})$ and $298.5 \mathrm{mg} / 100 \mathrm{~mL}$ after administration of $12 \mathrm{~mL} / \mathrm{kg}$. As the standard for alcohol poisoning is a BAC of $50-100 \mathrm{mg} /$ $100 \mathrm{~mL}$, the model was successful.

Tru Scan is a versatile and simple system to monitor open field activity. The MWM test is usually used to assess spatial learning and memory ability of experimental animals, and it has been a useful laboratory tool in behavioral neuroscience $(25,26)$. We used these tests to evaluate the effect of alcohol on the nervous system of experimental animals. A statistical analysis conducted in a large study of alcohol drinkers reported that physical activity among the excessive drinkers was significantly reduced (27). Alcohol poisoning may thus affect the CNS, which manifests as a decrease in movement function. In this study, the spontaneous movement recorded by the Tru Scan detection system confirmed the findings of Liangpunsakul et al. (27) in that ethanol poisoning led to a shorter spontaneous motion 

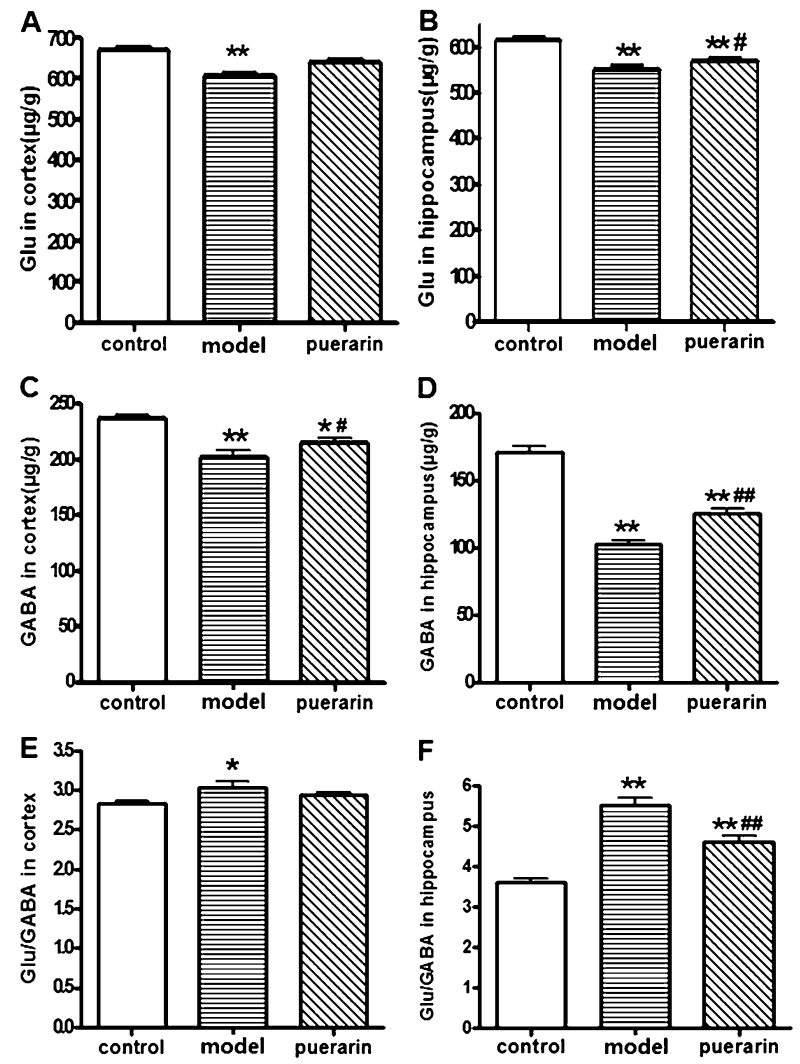

Figure 5. Detection of glutamic acid (Glu) and gamma amino butyric acid (GABA) by high-performance liquid chromatography (HPLC). A,B, Glu content of the cortex and hippocampus. $C, D$, GABA content of the cortex and hippocampus. $E, F$, Glu/GABA ratio in the cortex and hippocampus. ${ }^{*} \mathrm{P}<0.05$, ${ }^{* *} \mathrm{P}<0.01$ vs control group; ${ }^{\#} \mathrm{P}<0.05$, ${ }^{\# \#} \mathrm{P}<0.01$ vs model group (one-way ANOVA).

route, and that puerarin intervention inhibited the impairment by ethanol and promoted active spontaneous motion. Further, according to the results of the MWM test, chronic alcohol poisoning resulted in significant increases in the escape latency, escape distance, total swimming distance, and decrease in cross times. Therefore, chronic alcohol poisoning impaired spatial learning ability and memory. However, puerarin intervention significantly reversed these impairments as shown by decreases in escape latency, escape distance, total swimming distance, and increase in cross times. Moreover, mice in the control and puerarin groups appeared to choose a more effective route when searching for the platform. Therefore, ethanol poisoning impaired nervous system function, spatial learning ability, and memory, and puerarin intervention alleviated the damage caused by ethanol.

Microglia play a key, active role in regulating immune system functions in the CNS (28). In the absence of external stimulation, microglial cells remain in the resting
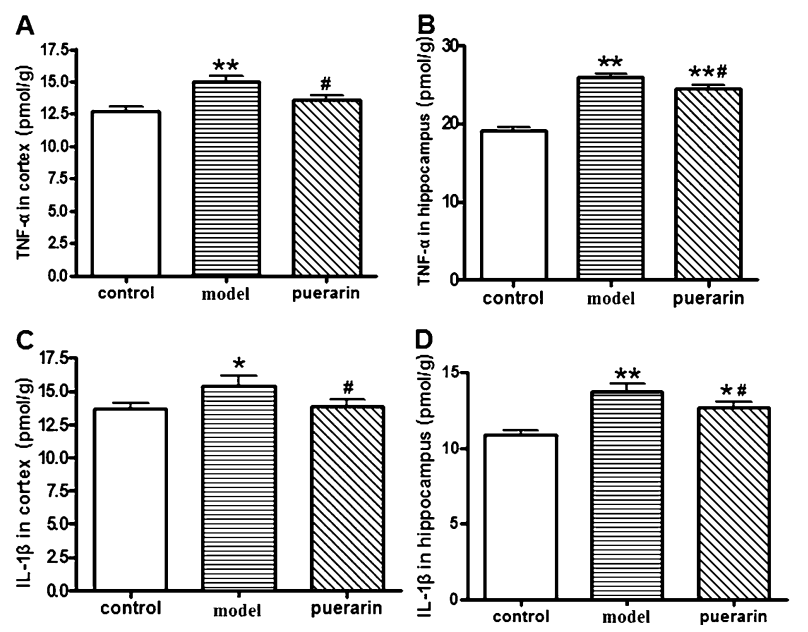

Figure 6. Detection of TNF- $\alpha$ and IL-1 $\beta$ by ELISA. $A, B$, Content of TNF- $\alpha$ in the cortex and hippocampus. $C, D$, Content of IL-1 $\beta$ in the cortex and hippocampus. ${ }^{*} \mathrm{P}<0.05$, ${ }^{\star \star} \mathrm{P}<0.01$ vs control group; ${ }^{\#} \mathrm{P}<0.05$ vs model group (one-way ANOVA).

state. After detecting an injury signal, their cell volume increases and the protuberances extending from their surface grow. These activated microglia, or brain macrophages, secrete neurotoxic inflammatory factors, such as TNF- $\alpha$ and IL $-1 \beta$, which can damage or kill neurons (Figure 7) (29-31). Previous studies indicate that alcohol poisoning can cause microglial activation, release of TNF- $\alpha$ and IL$1 \beta$, and damage to neurons (32). In this study, the numbers of microglia in both the hippocampal dentate gyrus and the cortex significantly increased, as did the levels of TNF- $\alpha$ and IL-1 $\beta$, and the number of neurons in the hippocampus significantly decreased. Puerarin intervention inhibited the changes in the numbers of microglia and neurons as well as secretion of TNF- $\alpha$ and IL-1 $\beta$. Therefore, we speculate that the protective effect of puerarin against damage caused by alcohol poisoning may be related mainly to the anti-inflammatory activity of puerarin (33), which reduces the activation of microglia and subsequent damage to or death of neurons, resulting in protection of spatial learning and memory ability.

Amino acid neurotransmitters in brain tissue, especially Glu and GABA, are closely related to CNS function. Excessive Glu can have toxic effects in the CNS (34). Although the levels of Glu and GABA were decreased by alcohol poisoning, increase of the Glu/GABA ratio indicates that the model produced a neurotransmitter imbalance. Relative increase of Glu has a toxic effect on neurons in the cortex and hippocampus, influencing the structure and function of the cortex and the hippocampus and causing impairment of spontaneous movement and spatial memory (35). Puerarin intervention decreased the Glu/GABA ratio in the cortex and hippocampus, especially in the cortex, where it decreased to near the control level. Therefore, 


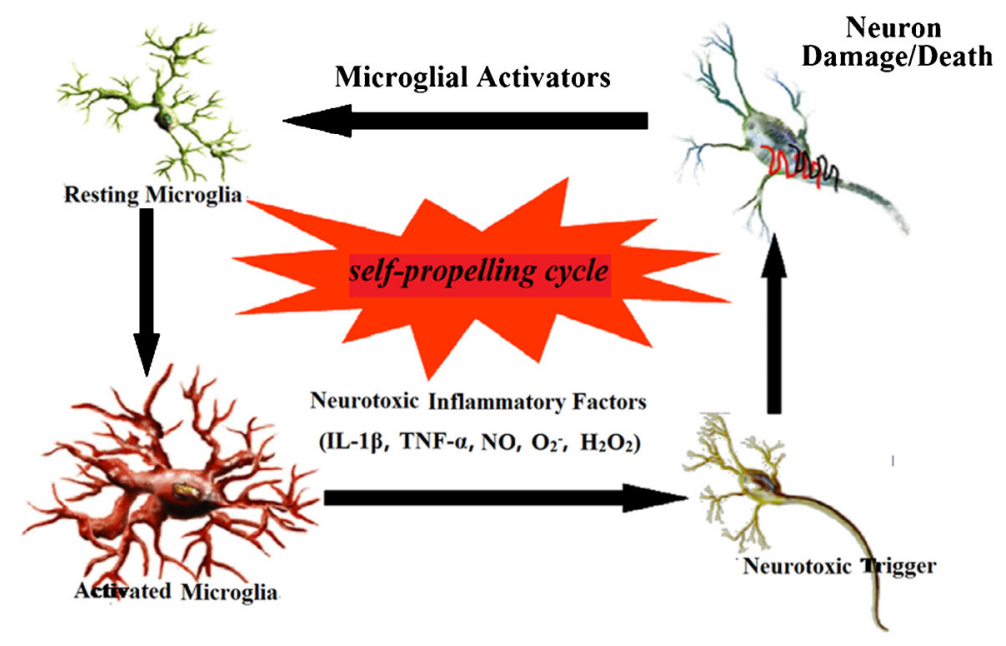

Figure 7. Diagram of changes in microglial appearance and function in the resting and activated states.

puerarin may have a regulatory effect on the balance of Glu and GABA, and further protect against the damage caused by chronic alcohol poisoning.

In conclusion, puerarin protected against damage of spatial learning and memory ability caused by chronic alcohol poisoning primarily because of anti-inflammatory activity and regulation of the balance of Glu and GABA. Further studies of its specific molecular mechanisms are needed.

\section{References}

1. Bakalkin G, Bazov I, Yakovleva T, Kuntic V, Sheedy D, Garrick T. S. 26.02 Alcoholism-associated molecular adaptations in endogenous opioids in brain neurocognitive circuits: human and animal correlates. Eur Neuropsychopharmacol 2008; 18: S194, doi: 10.1016/S0924-977X(08)70215-3.

2. McCully C. Goodbye, Mr. Wonderful: alcoholism, addiction and early recovery. London: Jessica Kingsley Publishers; 2004.

3. Matthews DB, Morrow AL. Effects of acute and chronic ethanol exposure on spatial cognitive processing and hippocampal function in the rat. Hippocampus 2000; 10: 122-130, doi: 10.1002/(SICI)1098-1063(2000)10:1<122::AIDHIPO13>3.0.CO;2-V.

4. Loeber S, Duka T, Welzel H, Nakovics H, Heinz A, Flor H, et al. Impairment of cognitive abilities and decision making after chronic use of alcohol: the impact of multiple detoxifications. Alcohol Alcohol 2009; 44: 372-381, doi: 10.1093/alcalc/ agp030.

5. Deik A, Saunders-Pullman R, Luciano MS. Substance abuse and movement disorders: complex interactions and comorbidities. Curr Drug Abuse Rev 2012; 5: 243-253, doi: $10.2174 / 1874473711205030243$.

6. Vetreno RP, Hall JM, Savage LM. Alcohol-related amnesia and dementia: animal models have revealed the contributions of different etiological factors on neuropathology, neurochemical dysfunction and cognitive impairment. Neurobiol Learn Mem 2011; 96: 596-608, doi: 10.1016/j.nIm.2011.01.003.

7. Rehm J, Mathers C, Popova S, Thavorncharoensap M, Teerawattananon Y, Patra J. Global burden of disease and

\section{Acknowledgments}

The authors appreciate the help by the Key Laboratory for Neurodegenerative Disorders of the Ministry of Education, Capital Medical University. Research supported by Shandong Province Natural Science Foundation of China (Shuqin Cui, no. ZR2010CL018, ZR2014HL039; Xiangling Gu, no. ZR2010BL001).

injury and economic cost attributable to alcohol use and alcohol-use disorders. Lancet 2009; 373: 2223-2233, doi: 10.1016/S0140-6736(09)60746-7.

8. Hensley K, Butterfield DA, Hall N, Cole P, Subramaniam R, Mark $R$, et al. Reactive oxygen species as causal agents in the neurotoxicity of the Alzheimer's disease-associated amyloid beta peptide. Ann N Y Acad Sci 1996; 786: 120134, doi: 10.1111/j.1749-6632.1996.tb39057.x.

9. Poeggeler B, Reiter RJ, Tan DX, Chen LD, Manchester LC. Melatonin, hydroxyl radical-mediated oxidative damage, and aging: a hypothesis. J Pineal Res 1993; 14: 151-168, doi: 10.1111/j.1600-079X.1993.tb00498.x.

10. Cassarino DS, Fall CP, Swerdlow RH, Smith TS, Halvorsen EM, Miller SW, et al. Elevated reactive oxygen species and antioxidant enzyme activities in animal and cellular models of Parkinson's disease. Biochim Biophys Acta 1997; 1362: 77-86, doi: 10.1016/ S0925-4439(97)00070-7.

11. Kedziora J, Bartosz G. Down's syndrome: a pathology involving the lack of balance of reactive oxygen species. Free Radic Biol Med 1988; 4: 317-330, doi: 10.1016/08915849(88)90052-4.

12. Gonenc S, Uysal N, Acikgoz O, Kayatekin BM, Sonmez A, Kiray M, et al. Effects of melatonin on oxidative stress and spatial memory impairment induced by acute ethanol treatment in rats. Physiol Res 2005; 54: 341-348.

13. Hashemi Nosrat Abadi T, Vaghef L, Babri S, Mahmood-Alilo $M$, Beirami M. Effects of different exercise protocols on ethanol-induced spatial memory impairment in adult male 
rats. Alcohol 2013; 47: 309-316, doi: 10.1016/j.alcohol.2013. 01.008.

14. Yu Z, Zhang G, Zhao H, Lu J. Effect of puerariae isoflavone on memory in mice. J China Pharm Univ 1996; 28: 350-353.

15. Dong LP, Wang TY. Effects of puerarin against glutamate excitotoxicity on cultured mouse cerebral cortical neurons. Zhongguo Yao Li Xue Bao 1998; 19: 339-342.

16. $\mathrm{Xu} \mathrm{X,} \mathrm{Zheng} X$. Potential involvement of calcium and nitric oxide in protective effects of puerarin on oxygen-glucose deprivation in cultured hippocampal neurons. J Ethnopharmacol 2007; 113: 421-426, doi: 10.1016/j.jep.2007.06.012.

17. Yao W, Sun K, Mu H, Liang N, Liu Y, Yao C, et al. Preparation and characterization of puerarin-dendrimer complexes as an ocular drug delivery system. Drug Dev Ind Pharm 2010; 36 : 1027-1035, doi: 10.3109/03639041003610799.

18. Sun J, Tan BK, Huang SH, Whiteman M, Zhu YZ. Effects of natural products on ischemic heart diseases and cardiovascular system. Acta Pharmacol Sin 2002; 23: 1142-1151.

19. Wu L, Qiao H, Li Y, Li L. Protective roles of puerarin and Danshensu on acute ischemic myocardial injury in rats. Phytomedicine 2007; 14: 652-658, doi: 10.1016/j.phymed. 2007.07.060.

20. Shi WG, Qu L, Wang JW. [Study on interventing effect of puerarin on insulin resistance in patients with coronary heart disease]. Zhongguo Zhong Xi Yi Jie He Za Zhi 2002; 22: 21-24.

21. Zhong $Z$, Zeng $\mathrm{T}$, Xie $\mathrm{K}$, Zhang $\mathrm{C}$, Chen J, Bi $\mathrm{Y}$, et al. Elevation of 4-hydroxynonenal and malondialdehyde modified protein levels in cerebral cortex with cognitive dysfunction in rats exposed to 1-bromopropane. Toxicology 2013; 306: 16-23, doi: 10.1016/j.tox.2013.01.022.

22. Room R. WHO European Ministerial Conference on Young People and Alcohol. Addiction 2004; 96: 787-788.

23. Riley ML. WHO to meet beverage company representatives to discuss health-related alcohol issues. Geneva: World Health Organization; 2003.

24. Cagetti E, Pinna G, Guidotti A, Baicy K, Olsen RW. Chronic intermittent ethanol $(\mathrm{CIE})$ administration in rats decreases levels of neurosteroids in hippocampus, accompanied by altered behavioral responses to neurosteroids and memory function. Neuropharmacology 2004; 46: 570-579, doi: 10.1016/j.neuropharm.2003.10.001.

25. Vorhees CV, Williams MT. Morris water maze: procedures for assessing spatial and related forms of learning and memory. Nat Protoc 2006; 1: 848-858, doi: 10.1038/nprot.2006.116.

26. D'Hooge R, De Deyn PP. Applications of the Morris water maze in the study of learning and memory. Brain Res Brain Res Rev 2001; 36: 60-90, doi: 10.1016/S0165-0173(01) 00067-4.

27. Liangpunsakul S, Crabb DW, Qi R. Relationship among alcohol intake, body fat, and physical activity: a populationbased study. Ann Epidemiol 2010; 20: 670-675, doi: 10.1016/ j.annepidem.2010.05.014.

28. Stoll G, Jander S. The role of microglia and macrophages in the pathophysiology of the CNS. Prog Neurobiol 1999; 58: 233-247, doi: 10.1016/S0301-0082(98)00083-5.

29. Streit WJ, Walter SA, Pennell NA. Reactive microgliosis. Prog Neurobiol 1999; 57: 563-581, doi: 10.1016/S03010082(98)00069-0.

30. Sullivan EV, Zahr NM. Neuroinflammation as a neurotoxic mechanism in alcoholism: commentary on "Increased MCP-1 and microglia in various regions of human alcoholic brain". Exp Neurol 2008; 213: 10-17, doi: 10.1016/j.expneurol.2008.05.016.

31. Ghoshal A, Das S, Ghosh S, Mishra MK, Sharma V, Koli P, et al. Proinflammatory mediators released by activated microglia induces neuronal death in Japanese encephalitis. Glia 2007; 55: 483-496, doi: 10.1002/glia.20474.

32. He J, Crews FT. Increased MCP-1 and microglia in various regions of the human alcoholic brain. Exp Neurol 2008; 210: 349-358, doi: 10.1016/j.expneurol.2007.11.017.

33. Xiao C, Li J, Dong X, He X, Niu X, Liu C, et al. Anti-oxidative and TNF-alpha suppressive activities of puerarin derivative (4AC) in RAW264.7 cells and collagen-induced arthritic rats. Eur J Pharmacol 2011; 666: 242-250, doi: 10.1016/j.ejphar. 2011.05.061.

34. Choi DW. Bench to bedside: the glutamate connection. Science 1992; 258: 241-243, doi: 10.1126/science.1357748.

35. White AM. What happened? Alcohol, memory blackouts, and the brain. Alcohol Res Health 2003; 27: 186-196. 